\title{
Arsenic exposure linked to diabetes risk in Canada
}

A rsenic is associated with a significantly increased risk of type II diabetes and prediabetes, according a study based on crossCanada biomonitoring data. This is the first time this emerging link, shown in studies from many parts of the world, has been tested here.

The study, published in June in Health Promotion and Chronic Disease Prevention in Canada, was drawn from cycle one of human biomonitoring of environmental chemicals in Canada, part of the Canadian Health Measures Survey conducted by Statistics Canada, in collaboration with Health Canada and the Public Health Agency of Canada, in 2007-2009. Data from cycle three (Jan. 2012-Dec. 2013) were released July 15 . According to an email from Health Canada, "there are no statistically significant differences in the levels measured" for arsenic among the three cycles of the survey data.

The study found that the highest quartile of arsenic exposure, measured in urine samples, is above rates that could be accounted for by a concentration in $0.01 \mathrm{mg} / \mathrm{L}$ of drinking water, the maximum acceptable concentration according to Canadian guidelines, explained the study's principal author, Dr. Solange Feseke Keboya of Centre de recherche $\mathrm{du}$ Centre hospitalier universitaire de Québec in Quebec City.

Arsenic exposure in the highest quartile approximately doubled the risk of type II diabetes (odds ratio just over 2) and prediabetes (odds ratio 1.8) compared with exposure in the lowest quartile. (Diabetes was defined as a serum glucose level of $126 \mathrm{mg} / \mathrm{dL}$ [7 mmol/L] or more or glycated hemoglobin of $6.5 \%$ or more, and prediabetes as a serum glucose level of $100-125 \mathrm{mg} / \mathrm{dL}$ [5.6-6.9 $\mathrm{mmol} / \mathrm{L}$ ] or glycated hemoglobin of $5.7 \%-6.4 \%$.)

"This is not a study that can show causality," cautioned Feseke Keboya, speaking in French. "It is difficult to confirm that arsenic leads to diabetes." However, she says studies around the world,

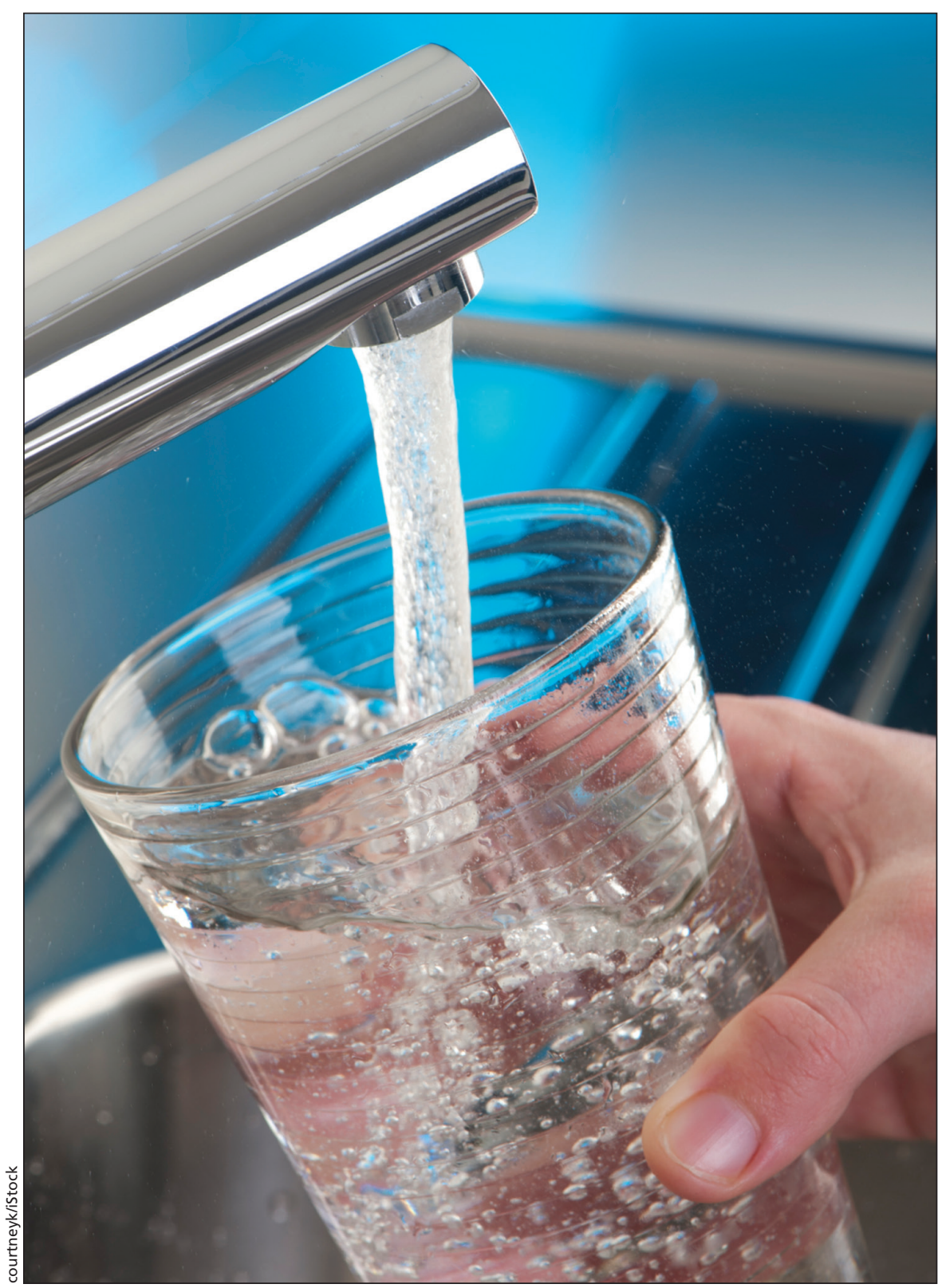

Naturally occurring arsenic is often found in groundwater, which is treated in urban areas, but well water in some regions can contain high levels.

including the United States and regions of Mexico where arsenic exposure is low, have also found the association.

The analysis adjusted for many factors that could affect diabetes risk, including body mass index, education level, age, sex, smoking and alcohol drinking in a representative sample of approximately 3517 Canadians aged 20 to 79 . The study also excluded participants who reported high consumption of fish, shellfish and other seafood. These food sources are known to bioaccumulate arsenic, but in organic forms thought to be less toxic, although this thinking is changing, said Feseke Keboya. Fish and seafood eaters were excluded in order to capture mainly inorganic arsenic. 
Arsenic occurs naturally and is often found in groundwater. Feseke Keboya said tap water is normally treated to bring levels within regulations, but well water in some regions can pose a danger of high levels. Health Canada states that arsenic is also used in some manufacturing processes, and therefore can be found in effluent. However, in most parts of Canada, Feseke Keboya said, the main source is food. According to Health Canada, arsenic is found at very low levels in meat and poultry, milk and dairy products, bakery goods and cereals, vegetables, and fruits and fruit juices.

Arsenic's toxic effects are well known. Acute exposure to arsenic can cause serious health effects, including skin problems, nausea, diarrhea, vomiting and numbness in hands and feet. Long-term exposure even at lower levels can cause lung, bladder and liver cancer.

Research is underway to understand how arsenic could lead to diabetes. "The pathology is very complex," explained Feseke Keboya. It is believed that arsenic acts through the liver, damaging cells, to affect insulin regulation and lead to hyperglycemia.

Although this study excluded participants who ate fish and seafood frequently - several times per week - this is a main source of arsenic in the Canadian diet, said Feseke Keboya. Until recently, the organic forms of arsenic found in fish and seafood were thought to be less toxic, but Feseke Keboya points to recent studies showing that some organic arsenic compounds are carcinogenic, and they have recently been classified as such by the International Agency for Research on Cancer. Although the effect of organic forms of arsenic on diabetes is less clear, Feseke Keboya emphasized, "all forms are dangerous." - Carolyn Brown, Ottawa

CMAJ 2015. DOI:10.1503/cmaj.109-5150 